\title{
When intensity of deltamethrin resistance in Anopheles gambiae s.l. leads to loss of Long Lasting Insecticidal Nets bio-efficacy: a case study in north Cameroon
}

Josiane Etang ${ }^{1,2^{*}}$, Cédric Pennetier ${ }^{3,4}$, Michael Piameu $^{5}$, Aziz Bouraima ${ }^{6}$, Fabrice Chandre ${ }^{3}$, Parfait Awono-Ambene ${ }^{1}$, Coosemans Marc ${ }^{7,8}$ and Vincent Corbel ${ }^{3,9}$

\begin{abstract}
Background: In Cameroon, insecticide resistance in Anopheles (An.) gambiae s.l. has been reported in several foci, prompting further investigations on associated patterns of Long-Lasting Insecticidal Nets (LLINs) bio-efficacy. The current study, conducted from June to August 2011, explored the intensity of deltamethrin resistance in An. gambiae s.l. from Pitoa and its impact on the residual bio-efficacy of LifeNet, a LLIN with deltamethrin incorporated into polypropylene nets (PND).

Methods: Two-four days old females An. gambiae s.l. reared from larval collections in Pitoa were tested for susceptibility to DDT, permethrin and deltamethrin, using standard World Health Organization (WHO) tube assays. Intensity of deltamethrin resistance was explored using WHO tube assays, but across six working concentrations from $0.001 \%$ to $0.5 \%$. Bio-efficacy of unwashed and washed PND was assessed using WHO cone test. Species identification and kdr 1014 genotyping were performed on mosquito samples that were not exposed to insecticides, using PCR-RFLP and HOLA methods respectively. The Kisumu reference susceptible strain of An. gambiae s.s. was used for comparisons.

Results: A total of 1895 An. gambiae s.l. specimens from Pitoa were used for resistance and PND bio-efficacy testing. This mosquito population was resistant to DDT, permethrin and deltamethrin, with 18-40 min knockdown times for $50 \%$ of tested mosquitoes and 59-77 \% mortality. Deltamethrin Resistance Ratio compared with the Kisumu strain was estimated at $\geq 500$ fold. LifeNets were effective against the susceptible Kisumu (100 \% knockdown $\left(K_{60 m i n}\right)$ and mortality) and the resistant Pitoa samples (95 \% KD 60 min, 83-95 \% mortality). However, the bio-efficacy gradually dropped against the Pitoa samples when nets were washed $\left(X^{2}=35.887, \mathrm{df}=8, p<0.001\right)$, and fell under the WHO efficacy threshold ( $80 \%$ mortality and/or $95 \% \mathrm{KD}_{60 \mathrm{~min}}$ ) between 10 and 15 washes. The Pitoa samples were composed of three sibling species: An. arabiensis (132/154, $86 \%)$, An. coluzzii (19/154, $12 \%)$ and An. gambiae s.s. (3/154, $2 \%)$. The kdr L1014F allele was found only in An. coluzzii ( $\left.N_{\text {positive }}=13 / 19\right)$, at $34 \%$ frequency and heterozygote stage. No specimen carried the $k d r$ L1014S allele.
\end{abstract}

Conclusions: The current study showed that LifeNet might still offer some protection against the resistant An. gambiae s.l. population from Pitoa, provided appropriate dose of insecticide is available on the nets.

Keywords: Deltamethrin, Anopheles gambiae s.l, Malaria, Resistance intensity, Long-Lasting Insecticidal Nets, Vector control

\footnotetext{
* Correspondence: josyet2@gmail.com

1 Institut de Recherche de Yaoundé (IRY), Organisation de Coordination pour

la lutte contre les Endémies en Afrique Centrale (OCEAC), B.P. 288, Yaoundé, Cameroun

${ }^{2}$ Faculty of Medicine and Pharmaceutical Sciences, University of Douala,

P.O. Box 2701, Douala, Cameroon

Full list of author information is available at the end of the article
} 


\section{Background}

Insecticide resistance in malaria vectors is a growing issue that jeopardizes efforts toward malaria elimination, since the general use of insecticides in Indoor Residual Spraying (IRS) or Long-Lasting Insecticidal Nets (LLINs) constitutes the only means of mass prevention of the disease [1]. Resistance is of particular concern in subSaharan Africa, where DDT and pyrethroid resistance is widespread in the major malaria vector species, i.e. those of the Anopheles (An.) gambiae complex and the $A n$. funestus group [2, 3].

In Cameroon, malaria annually accounts for 35 to $40 \%$ of deaths in health facilities, 40 to $45 \%$ of outpatient consultations, and $30 \%$ of hospitalisations. It is also responsible for $26 \%$ of job and school absenteeism and $40 \%$ of health spending in homes [4]. Malaria control efforts have been intensified over the last five years, and vector control is highly prioritized in the national strategic plan for malaria control [5]. Over eight and half million LLINs were distributed for free to the general population in 2011, covering at least $80 \%$ of pregnant women and children under five years old. The National Malaria Control Programme also aims to distribute twelve million LLINs in 2015 and 2016. The impact of these nationwide malaria vector control interventions on the disease burden, alongside implementation of rapid diagnostic tests and case management with Artemisinin combined therapy (ACT), is yet to be documented. Meanwhile, DDT and pyrethroid resistance is increasingly reported in three major malaria vectors belonging to the An. gambiae complex [6]: An. gambiae s.s, An. coluzzii and An. arabiensis [7-10]. Pyrethroid resistance in $A n$. gambiae s.l. was first reported in the Pitoa health district in the northern region of Cameroon. Subsequently, it has been reported in $A n$. gambiae s.l. from four more of the ten Regions (East, Centre, Littoral and West). The resistance has been conferred by two main mechanisms: (1) increased detoxification through high levels of mixed function oxidases, glutathione S-transferases or non-specific esterases and (2) alterations of insecticide target site via $k d r$ mutations in the gene coding for the voltage gated sodium channel. Some mosquito populations even display multiple insecticide resistance including both $k d r$ mutations and metabolic-based mechanisms $[10,11]$.

However, little is known about the magnitude and implications of insecticide resistance at the operational level, since many confounding factors make the interpretation of trial outcomes difficult [12]. Until recently, pyrethroid resistance based on $k d r$ mutations in $A n$. gambiae from Côte d'Ivoire [13, 14] did not adversely affect the effectiveness of pyrethroid-treated bed nets in terms of reduction of man-vector contact and asymptomatic infections, and in terms of protection against malaria attacks. Conversely, studies conducted in southern Benin showed a lower effectiveness of LLINs against resistant mosquitoes, with neither asymptomatic infections, nor malaria attacks, being reduced [15-17].

Considering historic and contemporary data on $A n$. gambiae s.l. vector species and their resistance phenotypes, malaria burden and intervention coverage, the Pitoa health district in Cameroon appears as an ideal location for further investigations of the magnitude of insecticide resistance and its effects on conventional vector control tools. Due to the complexity of the malaria transmission system and the rapid increase of An. gambiae s.l. resistance to insecticides in Pitoa, achieving malaria elimination is anticipated to be difficult in this health district. Malaria incidence increased from $54.4 \%$ in 2002 [18] to $61.5 \%$ in 2011 [Etang and Bigoga, personal communication]. This incidence is among the highest of the twelve health districts of northern Cameroon region, including Garoua Nord and Garoua Centre health districts (around $46 \%$ malaria incidence).

The objective of this study was to evaluate Bayer LifeNet (batch 2010-004024) under laboratory conditions and assess its intrinsic bio-efficacy and wash resistance against the pyrethroid-resistant strain of An. gambiae s.l. from Pitoa. For this purpose, we updated the status of DDT and pyrethroid resistance in An. gambiae s.l. population from Pitoa as defined by the WHO standard criteria. Dose-response tests subsequently revealed a noteworthy increase of deltamethrin resistance in this An. gambiae s.l. population compared with the Kisumu susceptible reference strain of An. gambiae s.s. After 10-15 washes, the deltamethrin on the LifeNet was no longer effective against the An. gambiae s.l population from Pitoa.

\section{Methods \\ Study site}

The study was conducted in the Pitoa health district $\left(09^{\circ} 23^{\prime} 31^{\prime \prime} \mathrm{N}, 13^{\circ} 30^{\prime} 09^{\prime \prime} \mathrm{E}\right)$, from June to August 2011. Pitoa is small city situated at about $15 \mathrm{~km}$ from Garoua town, which is the capital city of the northern region of Cameroon.

The epidemiology of malaria in Pitoa is particularly complex, owing to its agro-economic and climatic environments and the variety in parasite and vector species compositions [18]. This health district is surrounded by cotton growing fields in the savannah area (about 35 000 ha cultivated area). Malaria transmission is seasonal, with a sudden rise of new infections acquired during the rainy season (May-October). There is a peak transmission season (September-October) and a low transmission season (April-May). Malaria infection is essentially due to Plasmodium falciparum, with few $P$. malariae. Several Anopheles species have been incriminated as 
vectors, mainly those of the An. funestus group and $A n$. gambiae complex [18]. The dynamics of vector populations, parasite transmission and disease burden with the roll out of LLINs is currently under in-depth investigation [Etang and Bigoga, personal communication].

However, previous studies showed a moderate level of resistance to permethrin, deltamethrin and DDT in $A n$. gambiae s.l., mainly due to high oxidase and esterase activities $[8,9,11]$. Furthermore, the $k d r L 1014 F$ mutation was reported in the local population of An. gambiae s.s., although at very low frequency [19].

\section{Mosquitos collection and sampling}

An. gambiae s.l. larvae and pupae were collected from breeding sites in August 2011 and reared locally until adult emergence. Adult mosquitoes were identified using morphological identification reference keys $[20,21]$. Only females An. gambiae s.l. aged two-four days old were used for insecticide resistance and LifeNet bioefficacy testing.

\section{Resistance testing}

DDT and pyrethroid resistance was tested using the standard World Health Organization (WHO) susceptibility test procedures for adult mosquitoes. Tests were performed under ambient room temperature $\left(25-28{ }^{\circ} \mathrm{C}\right)$ and relative humidity of $70-80 \%$ [22].

Mosquitoes were exposed for one hour to diagnostic concentrations of DDT (4\%), deltamethrin $(0.05 \%)$ orpermethrin $(0.75 \%)$ on impregnated papers, purchased from University Sains, Malaysia. For each insecticide concentration, susceptibility bioassays were performed with five batches of 20-25 unfed females: four batches were exposed to insecticide-impregnated filter papers and one batch was exposed to untreated filter paper as a control. After one hour-long exposure, mosquitoes were transferred to holding tubes and provided with cotton pads soaked with $10 \%$ sugar solution. The number of mosquitoes knocked-down was recorded at five minute intervals during the one hour-long exposure and mortality was determined $24 \mathrm{~h}$ post exposure.

Resistance status was evaluated according to the WHO criteria [22], which classify mortality rates of less than $90 \%$ as indicative of resistance while those greater than $98 \%$ indicate susceptibility. Mortality rates between 90-98 \% suggest the possibility of resistance that needs to be verified.

Tests were concomitantly performed with the Kisumu susceptible reference strain of An. gambiae s.s. maintained in the OCEAC (Yaoundé, Cameroon) insectaries.

\section{Evaluation of resistance intensity}

The intensity of deltamethrin resistance in the Pitoa wild An. gambiae s.l. population was also assessed using
WHO standard tube test protocol for adult mosquitoes [22], with a range of 5 deltamethrin concentrations $(0.001,0.005,0.01,0.1$ and $0.5 \%)$, in addition to the classic diagnostic concentration $(0.05 \%)$.

Filter paper sheets were impregnated with technicalgrade deltamethrin (SIGMA ALDRICH) or control solution (acetone + silicon oil) by the research team, at least $24 \mathrm{~h}$ prior to use. A stock solution of $1 \%$ deltamethrin was prepared by mixing $204 \mathrm{mg}$ of the commercial product (98\%) with $20 \mathrm{ml}$ of acetone. Then, six working solutions of deltamethrin were prepared by serial dilution of the stock solution with acetone and silicon oil $(40 \mathrm{ml}$ silicon $+60 \mathrm{ml}$ acetone $=100 \mathrm{ml}$ dilution solution), to obtain $0.001,0.005,0.01,0.1$ and $0.5 \%$ working solutions respectively. Acetone acted as the solvent; silicon oil (SIGMA ALDRICH) served as the carrier for deltamethrin. For each deltamethrin concentration, four sheets of filter paper $\left(12 \times 15 \mathrm{~cm}\right.$ WhatmanN $\left.{ }^{\circ} 1\right)$ were impregnated with two millilitres of working solution (deltamethrin + acetone + silicon oil) each, using a micropipette.

A batch of four filter paper sheets was impregnated with acetone + silicon solution for use as control. The impregnated papers were allowed to dry on a wire fence for $24 \mathrm{~h}$ at room temperature $\left(27-30{ }^{\circ} \mathrm{C}\right)$, then were wrapped in aluminum foil and stored at $4{ }^{\circ} \mathrm{C}$ until the date of the test.

For each of the six deltamethrin concentrations, four to five batches of 20-25 mosquitoes were exposed for one hour to deltamethrin-impregnated papers. The number of mosquitoes knocked down was recorded at five minute intervals during the one hour-long exposure and mortality was determined $24 \mathrm{~h}$ post exposure.

Tests were concomitantly performed with same range of deltamethrin concentrations on the Pitoa wild mosquito samples and laboratory samples of the Kisumu susceptible reference strain of An. gambiae s.s. maintained in the OCEAC (Yaoundé, Cameroon) insectaries. The increase in tolerance or resistance level in adult mosquitoes of the Pitoa population was calculated by comparing their knock-down times and mortality with those of the Kisumu susceptible strain.

\section{Assessment of long lasting insecticidal nets bio-efficacy Long lasting insecticidal nets and washing procedures}

Long Lasting Insecticidal Nets used in this study were from LifeNet brand, a WHO interim recommended LLIN. According to the manufacturer (Bayer CropScience), the fabric is made of polypropylene and has a deltamethrin load of $8.5 \mathrm{a} . \mathrm{i} \mathrm{g} / \mathrm{kg}$ for 100 denier net fabric (PND), resulting in a deltamethrin concentration of $340 \mathrm{mg}$ a.i. $/ \mathrm{m}^{2}$ incorporated into the fibre material.

A $30 \mathrm{~cm} \times 30 \mathrm{~cm}$ piece of polyester netting (100 denier) treated with deltamethrin at $25 \mathrm{mg}$ a.i $/ \mathrm{m}^{2}$ (hand-dipped PRD) by the research team using the dipping method was 
used as positive control and untreated polyester nets as negative control.

For this study, Bayer provided samples of LifeNet wrapped in aluminium foil (washed and unwashed), one piece of $30 \mathrm{~cm} \times 30 \mathrm{~cm}$ netting for each of $0,5,10,15,20$, 25,30 or 35 washes. LLIN samples were kept in aluminum foil and stored at room temperature $\left(27-30{ }^{\circ} \mathrm{C}\right)$ until the date of the test. Details of standard washing and bioassays are provided in the $\mathrm{WHO}$ guidelines for testing and evaluation of LLINs [23]. Briefly, washing included placing a net sample into a 1-1 beaker in which $2 \mathrm{~g} / \mathrm{l}$ soap (savon de Marseille, $\mathrm{pH} 10-11$ ) was fully dissolved into $0.5 \mathrm{l}$ deionized water. The beakers were introduced into a $30{ }^{\circ} \mathrm{C}$ water-bath and shaken for $10 \mathrm{~min}$ at 155 movements per minute. The samples were then removed, rinsed twice for $10 \mathrm{~min}$ in clean, deionized water under the same shaking conditions as above, dried at room temperature and stored at $30^{\circ} \mathrm{C}$ in the dark between washes.

\section{Cone test}

Bio-efficacy of LifeNet against the Pitoa field collected and the Kisumu laboratory reared samples of $A n$. gambiae s.l. was assessed using the WHO cone test to measure knock-down and mortality of mosquitoes after contact with nets [23]. Batches of five non-blood fed, two to four days old An. gambiae s.l. females were exposed for three minutes to net samples and held for $24 \mathrm{~h}$ with access to sugar solution. One hundred mosquitoes ( 5 mosquitoes $\times 20$ cones) were exposed to each of the net samples and results pooled for analysis. Mosquitoes exposed to hand-dipped PRD and untreated nets were positive and negative controls, respectively. Bioassays were carried out at $27 \pm 2{ }^{\circ} \mathrm{C}$ and $75 \pm 10 \%$ $\mathrm{RH}$. Knock-down was measured at 60 minutes post- exposure and mortality after $24 \mathrm{~h}$.

The number of washes and bioassays performed with each of the Kisumu susceptible and the Pitoa resistant strains of An. gambiae s.l. are summarized as followed:

- one PND (LifeNet) sample $x$ five mosquito replicates $\mathrm{x}$ height configurations $(0,5,10,15,20,25,30$ and 35 washes) $=$ forty cone tests (eight hundred females);

- one PRD (hand treated with deltamethrin using the dipping method) sample $x$ five replicates $x$ one configuration = five cone tests (one hundred females);

- one untreated sample $\mathrm{x}$ five replicates $\mathrm{x}$ one configuration $=$ five cone tests (one hundred females).

\section{Species identification and $k d r$ genotyping}

Mosquitoes used as control during susceptibility, resistance intensity and cone tests were used for species identification and $k d r 1014$ genotyping, in order to estimate the frequencies of $k d r$ alleles in the tested samples. DNA was extracted from each specimen using the method of
Collins et al. [24], and each individual was identified to the species level using PCR-RFLP [25]. This method allows simultaneous identification of the species of the An. gambiae complex. Alleles at the kdr1014 locus were genotyped using hot oligonucleotide ligation assay (HOLA) as described by Lynd et al. [26].

\section{Data analysis}

The knock-down times for 50 and $95 \%\left(\mathrm{KDT}_{50}\right.$ and $\left.\mathrm{KDT}_{95}\right)$ mosquitoes during exposure to insecticide impregnated papers in susceptibility tests were estimated using a log-time probit model [27]. The log-probit analysis was performed using the WIN DL (version $2.0,1999)$ software. The $\mathrm{KDT}_{50}$ recorded from fieldcollected mosquitoes were compared with that of the Kisumu reference susceptible strain of $A n$. gambiae by estimates of $\mathrm{KDT}_{50}$ Ratios $\left(\mathrm{KDT}_{50} \mathrm{R}\right)$.

The minimum plausible Resistance Ratio (RR) was estimated based on lethal concentration for $100 \%$ mosquitoes of the Pitoa samples and that of the Kisumu susceptible samples as followed: $\mathrm{RR}=\mathrm{LC}_{100}$ Pitoa $/ \mathrm{LC} \mathrm{C}_{100}$ Kisumu.

Bio-efficacy of mosquito nets was estimated by means of mosquito knock-down rates 60 min post exposure to treated-nets $\left(\mathrm{KD}_{60 \mathrm{~min}}\right)$ and mortality rates $24 \mathrm{~h}$ post exposure via cone test. The number of washes generating mortality and/or $\mathrm{KD}_{60 \mathrm{~min}}$ above the cut-off point (more than $80 \%$ mortality after $24 \mathrm{~h}$ and/or above $95 \%$ $\left.\mathrm{KD}_{60 \mathrm{~min}}\right)$ was reported for each strain. The variations of $\mathrm{KD}_{60 \mathrm{~min}}$ and mortality rates in the Pitoa An. gambiae s.l population in contact with washed LLINs were analysed using the Pearson's Chi-square test of independence, with R software (Version 2.15.2, R Development Core Team 2005).

\section{Results}

\section{Status of DDT and pyrethroid resistance}

A total of 314 An. gambiae s.l. females from Pitoa were used for susceptibility tests: 294 exposed to insecticides (4 \% DDT, $0.75 \%$ permethrin or $0.05 \%$ deltamethrin) and 20 control. Also, 278 An. gambiae s.s. females from the Kisumu strain were tested: 258 exposed to insecticides and 20 control. The number of mosquitoes exposed to a given diagnostic concentration of insecticide varied from 85 to 110 . The recorded knock-down (KD) times and mortality rates are given in Table 1.

Knock-down and mortality rates were $0-3 \%$ in control mosquitoes from either the Kisumu strain or the Pitoa wild population.

The Kisumu strain was fully susceptible to the three insecticides. The knock-down times for $50 \%\left(\mathrm{KDT}_{50}\right)$ mosquitoes were $20 \mathrm{~min}$ in contact with DDT and around $10 \mathrm{~min}$ with permethrin or deltamethrin. For the three insecticides, $\mathrm{KDT}_{95}$ were less than $32 \mathrm{~min}$ and 
Table 1 Knock-down times for 50 and $95 \%$ of the Kisumu susceptible strain and the Pitoa Anopheles gambiae s.l. population to DDT, permethrin and deltamethrin diagnostic concentrations

\begin{tabular}{|c|c|c|c|c|c|c|c|c|c|}
\hline \multirow{2}{*}{$\begin{array}{l}\text { Diagnostic } \\
\text { concentrations }\end{array}$} & \multicolumn{4}{|c|}{ Kisumu strain } & \multicolumn{5}{|c|}{ Pitoa popualtion } \\
\hline & $\mathrm{N}$ & $\mathrm{kdT}_{50}[\mathrm{Cl}](\mathrm{min})$ & $\mathrm{kdT}_{95}[\mathrm{Cl}]$ (min) & Mortality (\%) & $\mathrm{N}$ & $\mathrm{kdT}_{50}[\mathrm{Cl}](\mathrm{min})$ & $\mathrm{kdT}_{95}[\mathrm{Cl}](\mathrm{min})$ & $\mathrm{KDT}_{50} \mathrm{R}$ & Mortality (\%) \\
\hline $4 \%$ DDT & 80 & $19.8[17.5-21.3]$ & $31.2[28.5-33.1]$ & 100 & 85 & $40.3[38.1-43.1]$ & $>60$ & 2.0 & 76.5 \\
\hline $0.05 \%$ Deltamethrin & 91 & $10.1[8.2-12.8]$ & $22.2[20.1-24.9]$ & 100 & 110 & 19.6 [17.9-21.3] & $37.6[33.2-39.5]$ & 1.9 & 59.1 \\
\hline 0.75 \% Permethrin & 87 & 9.1 [7.5-11.5] & 21.4 [19.2-23.3] & 100 & 99 & 21.9 [18.7-23.5] & 45.3 [39.8-48.5] & 2.4 & 72.7 \\
\hline
\end{tabular}

$N$ Sample size, $\mathrm{Cl}$ confidence interval at $95 \%, k d T_{50}$ Knock-down times for $50 \%$ of exposed mosquitoes, $K D T_{95}$ Knock-down times for $95 \%$ of exposed mosquitoes, $K D T_{50} R$ Ratio $\mathrm{KDT}_{50} \mathrm{Pitoa} / \mathrm{KDT}_{50} \mathrm{Kisumu}$, min time in minutes

$100 \%$ exposed mosquitoes were dead at $24 \mathrm{~h}$ post exposure.

Conversely, the Pitoa population of An. gambiae s.l. was found to be resistant to the three insecticides. The $\mathrm{KDT}_{50}$ was around 40, 20 and 22 min with DDT, deltamethrin and permethrin respectively. The $\mathrm{KDT}_{50}$ Ratio compared to the Kisumu strain ranged from 1.9 to 2.4 fold. At the end of the 60-min exposure time, knockdown rate did not reach $95 \%$ for DDT. Meanwhile, the $\mathrm{KDT}_{95}$ were around 38 and 45 min for deltamethrin and permethrin respectively. For the three insecticides, the mortality rates at $24 \mathrm{~h}$ ranged from $59 \%$ to $77 \%$.

\section{Resistance intensity}

A total of 575 females An. gambiae s.l. from Pitoa were used for resistance intensity test, including 514 specimens exposed to a range of 6 deltamethrin concentrations $(0.001 \%$ to $0.5 \%)$ and 61 specimens used as control. Also, 585 females An. gambiae s.s. from the Kisumu strain were tested, including 520 specimens exposed to deltamethrin impregnated papers and 65 control specimens. The number of mosquitoes submitted to each one of the six deltamethrin concentration varied from 70 to 110 , depending on the number of larvae previously collected from the field and availability of twofour days females on the day of the test.

Resistance intensity was expressed as a dose-response effect in terms of $\mathrm{KDT}_{50}, \mathrm{KDT}_{95}$ and mortality rates to deltamethrin (Table 2). Knock-down and mortality rates of control mosquitoes ranged from $0 \%$ to $4 \%$ in both the Kisumu strain and the Pitoa population.

\section{Times of knock-down}

The $\mathrm{KDT}_{50}$ of the Kisumu strain decreased when the deltamethrin concentrations increased, ranging from $34.6 \mathrm{~min}$ at $0.001 \%$ to $0.8 \mathrm{~min}$ at $0.5 \%$. The $\mathrm{KDT}_{95}$ also decreased from $65.6 \mathrm{~min}$ to $6.6 \mathrm{~min}$. Between 0.005 and $0.01 \%$ deltamethrin concentrations, however, the knock-down time did not decrease $\left(\approx 18\right.$ min $\mathrm{KDT}_{50}, 26-27$ min $\left.\mathrm{KDT}_{95}\right)$.

With the Pitoa field collected mosquitoes, the $\mathrm{KDT}_{50}$ decreased from $>60 \mathrm{~min}$ at $0.001 \%$ deltamethrin to $7.0 \mathrm{~min}$ at $0.5 \%$ deltamethrin. The $\mathrm{KDT}_{95}$ also decreased from $>60 \mathrm{~min}$ to $17.2 \mathrm{~min}$.

The $\mathrm{KDT}_{50}$ Ratio $\left(\mathrm{KDT}_{50} \mathrm{R}\right)$ correspondingly increased from 2.2 to 8.7 when deltamethrin concentration was increased.

\section{Mortality rates}

Mortality rates of Kisumu and Pitoa An. gambiae s.l. samples in deltamethrin dose-response tests are given in Fig. 1.

The mortality rates of the Kisumu reference strain to the 6 targeted concentrations were constantly $100 \%$. This maximum mortality rate did not allow estimation of the lethal concentration for $50 \%$ mosquitoes $\left(\mathrm{LC}_{50}\right)$ of the Kisumu strain. Conversely, mortality rates of the Pitoa population increased from $19 \%$ to $100 \%$. The $\mathrm{LC}_{50}$ was estimated at $0.019 \%$ deltamethrin. The concentration

Table 2 Knock-down times for 50 and $95 \%$ of the Kisumu susceptible and the Pitoa resistant Anopheles gambiae s.l. strain to increased concentrations of deltamethrin on impregnated papers

\begin{tabular}{|c|c|c|c|c|c|c|c|}
\hline \multirow[t]{2}{*}{ Deltamethrin dosage (\%) } & \multicolumn{3}{|c|}{ Kisumu strain } & \multicolumn{4}{|c|}{ Pitoa strain } \\
\hline & $\mathrm{N}$ & $\mathrm{kdT}_{50}[\mathrm{Cl}](\mathrm{min})$ & $\mathrm{kdT}_{95}[\mathrm{Cl}](\mathrm{min})$ & $\mathrm{N}$ & $\mathrm{kdT}_{50}[\mathrm{Cl}(\mathrm{min})]$ & $\mathrm{kdT}_{95}[\mathrm{Cl}](\mathrm{min})$ & $\mathrm{KdT}_{50}$ ratio \\
\hline 0.001 & 80 & $34.6[12.2-42.1]$ & $>60$ & 78 & $>60$ & $>60$ & 2.2 \\
\hline 0.005 & 96 & 18.6 [17.9-19.4] & $26.1[24.4-28.9]$ & 67 & $48.3[40.7-60.2]$ & $>60$ & 2.5 \\
\hline 0.01 & 68 & $18.4[17.5-19.4]$ & $27.2[24.9-31.0]$ & 98 & $21.7[18.5-24.9]$ & $57.2[45.8-82.3]$ & 1.2 \\
\hline 0.05 & 90 & $3.9\left[5.9 \times 10^{-6}-2.6 \times 10^{6}\right]$ & $17.7\left[9.1 \times 10^{-3}-3.4 \times 10^{4}\right]$ & 110 & $17.8[15.9-19.6)$ & $59.3[52.0-69.9]$ & 4.6 \\
\hline 0.1 & 92 & $1.3\left[7.5 \times 10^{-5}-2.6 \times 10^{4}\right]$ & $9.3\left[0.06-1.4 \times 10^{3}\right]$ & 74 & $9.6[8.6-10.0]$ & $32.3[28.0-38.5]$ & 7.4 \\
\hline 0.5 & 94 & $0.8\left[1.8 \times 10^{-8}-3.7 \times 10^{7}\right]$ & $6.6\left[2.5 \times 10^{-4}-1.6 \times 10^{5}\right]$ & 87 & $7.0[4.0-9.0]$ & $17.2[14.1-24.3]$ & 8.7 \\
\hline
\end{tabular}

$N$ Sample size, $\mathrm{Cl}$ confidence interval at $95 \%$, kdT50 Knockdown times for $50 \%$ of exposed mosquitoes, $k d T_{95}$ Knockdown times for $95 \%$ of exposed mosquitoes, min time in minutes 


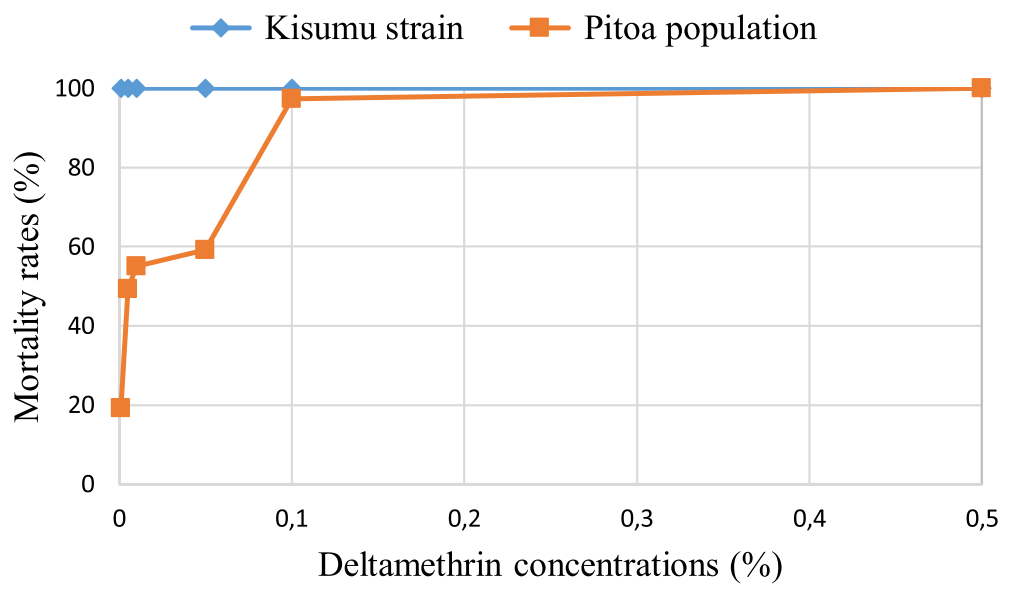

Fig. 1 Mortality rates of Kisumu and Pitoa Anopheles gambiae s.l. samples in deltamethrin dose-response test

required to kill $100 \%$ mosquitoes $(0.5 \%)$ was 10 fold higher than the resistance-discriminating dose for deltamethrin (0.05\%).

Although the $\mathrm{LC}_{50}$ of the Kisumu strain could not be estimated, its value was expected to be less than $0.001 \%$, i.e. the lowest deltamethrin concentration tested. Therefore, the Resistance Ratio (RR) of the Pitoa population was estimated higher than 500 -fold.

\section{Bio-efficacy of LifeNet}

The bio-efficacy of LifeNet (deltamethrin incorporated) was evaluated before $(0 \mathrm{x})$ and after an increasing number of washes: 5x, 10x, 15x, 20x, 25x, 30x, 35x. Approximately 100 mosquitoes were used to test each net type.

A total of 1006 females An. gambiae s.l. from Pitoa were used for cone tests, including 904 specimens exposed to unwashed and washed LifeNet (PND), 100 specimens exposed to positive control net (hand-dipped PRD) and 102 specimens exposed to negative control net (untreated net). Also, a total of 1006 females $A n$. gambiae s.l. from the Kisumu strain were used, including 904 specimens exposed to unwashed and washed LifeNet, 101 exposed to positive control (PRD) and 100 exposed to negative control net. The recorded knockdown and mortality rates are given in Fig. 2.

With untreated net, the knockdown and mortality rates of both susceptible (Kisumu strain) and fieldderived, resistant mosquito samples (Pitoa strain) were $0 \%$, confirming that there was no contamination during the experiments.

The hand-dipped PRD nets generated $100 \% \mathrm{KD}_{60 \text { min }}$ and mortality against the Kisumu strain, versus $86 \%$ $\mathrm{KD}_{60 \text { min }}$ and $48 \%$ mortality rates against the Pitoa strain, which corroborates Pitoa strain's deltamethrin resistance identified in the dose-response assay.

Unwashed and washed LifeNet generated $100 \%$ $\mathrm{KD}_{60 \mathrm{~min}}$ and mortality rates against the Kisumu strain.
Against the Pitoa strain, the $\mathrm{KD}_{60 \mathrm{~min}}$ was mostly higher than $95 \%$, even after 35 washes. The subsequent mortality rates were high with unwashed nets, as well as nets washed up to 10 times (83-95\%). Nevertheless, the number of washes had a significant influence on mosquito mortality rates $\left(X^{2}=35.887, \mathrm{df}=8, p<0.001\right)$, which gradually decreased from $95 \%$ with unwashed PRD to $32 \%$ with PRD washed 35 times. After 15 washes, the mortality rate dropped at $72 \%$, i.e. below the cut-off of LLINs efficacy.

\section{Species diversity and allelic frequencies at the $k d r$ 1014 locus}

A total of 183 An. gambiae s.l. specimens from Pitoa used as control (i.e. not exposed to insecticides) during susceptibility, resistance intensity and cone tests were submitted to species identification and $k d r$ L1014F and L1014S genotyping; 154 specimens were successfully analysed. Results are given in Table 3.

Three sibling species of the An.gambiae complex were identified in the Pitoa population. An. arabiensis was the predominant species, representing $86 \%$, followed by $A n$. coluzzii (12\%) and An. gambiae s.s. (2\%). The $k d r$ L1014F was found only in An. coluzzii, at $34 \%$ frequency, and only in theheterozygous state. The frequency of $k d r L 1014 F$ allele in An. gambiae s.l. overall was $4.2 \%$. The $k d r$ L1014S allele was not found in any of the tested specimens.

\section{Discussion}

In this study, we attempted to address the question of possible change in the biological activity of LLINs against the An. gambiae s.l. population from Pitoa in North Cameroon, which is known to be resistant to DDT and pyrethroid insecticides. The findings revealed that, under laboratory testing conditions, newly operating LifeNet LLINs were effective against the local 


\section{A KNOCK DOWN}

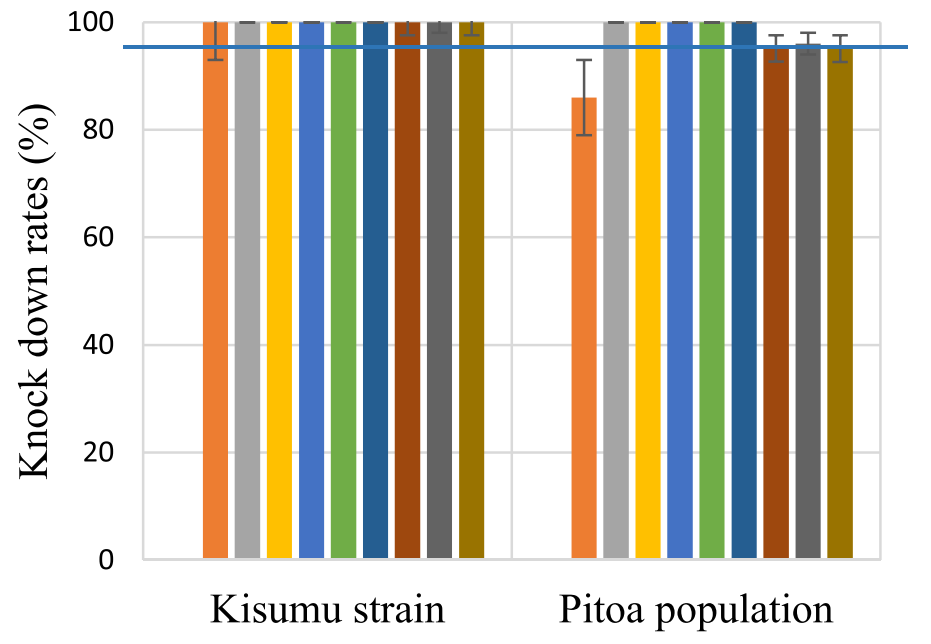

PRD 25mg/m2 OX

PND $0.85 \%$ OX

PND $0.85 \% 5 \mathrm{X}$

PND 0.85\% 10X

PND 0,85\% 15X

PND $0.85 \%$ 20X

DND $0.85 \% 25 \mathrm{X}$

PND $0.85 \% 30 \mathrm{X}$

PND $0.85 \% 35 X$

\section{B MORTALITY}

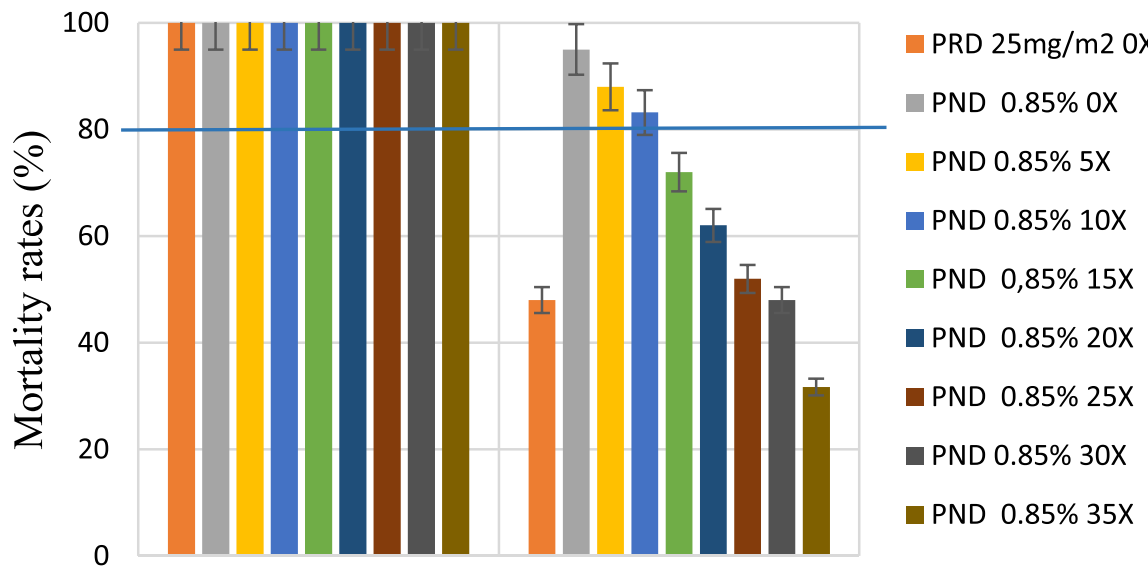

Kisumu strain Pitoa population

Fig. 2 Knock-down and mortality rates of Kisumu and Pitoa Anopheles gambiae s.l. samples to deltamethrin treated nets. a: knock-down rates of An. gambiae s.l. samples 60 min post exposure to insecticide treated nets; $\mathbf{b}$ : mortality rates of An. gambiae s.l. samples 24 h post exposure to insecticide treated nets; PRD: polyester nets impregnated with deltamethrin; PND: polypropylene nets incorporating delatmethrin; Blue lines indicate cut-off point of nets efficacy (95\% knock-down rate 60 min post exposure and/or $80 \%$ mortality rate 24 hours post exposure)

Table 3 Species diversity and kdr 1014 genotypes in Anopheles gambiae s.l. from Pitoa

\begin{tabular}{lllll}
\hline Species & $N(\%)$ & \multicolumn{4}{l}{$K d r$ genotypes } \\
\cline { 3 - 5 } & & $1014 \mathrm{~L}(\mathrm{~S})$ & $1014 \mathrm{~F}\left(\mathrm{R}_{\mathrm{w}} / \mathrm{S}\right)$ & $1014 \mathrm{~S}\left(\mathrm{R}_{\mathrm{e}}\right)$ \\
\hline An. coluzzii & $19(12)$ & 6 & 13 & 0 \\
An. gambiae & $3(2)$ & 3 & 0 & 0 \\
An. arabiensis & $132(86)$ & 132 & 0 & 0 \\
TOTAL $^{\mathrm{b}}$ & 154 & 141 & 13 & - \\
\hline
\end{tabular}

$N$ sample size, ${ }^{\mathrm{a} F r e q u e n c y}$ of $k d r 1014 \mathrm{~F}$ allele in An. coluzzii $=0.34,{ }^{\mathrm{b}}$ Frequency of $k d r 1014 \mathrm{~F}$ allele in overall An. gambiae s.l. $=0.042$ deltamethrin resistant An. gambiae s.l. population; but this bio-efficacy declined after nets were washed more than 10 times. By contrast, unwashed nets and nets washed up to 35 times were found effective against the Kisumu susceptible laboratory strain of An. gambiae s.s. The bio-efficacy of LifeNet against the Kisumu strain was consistent with the WHOPES criteria [23], in which "a LLIN would be expected to retain its biological activity for at least 20 standard washes under laboratory conditions" ( $>80 \%$ mortality and/or $>95 \%$ knock-down). The intensity of deltamethrin resistance in field samples of An. gambiae s.l. likely played a major role in the gradual decrease of LifeNet bio-efficacy after serial 
washing, emphasizing the importance of assessing the magnitude of insecticide resistance and subsequent impact on vector control efficacy.

Until recently, insecticide resistance testing has been focused on discriminating doses of insecticides using either WHO tube Assay or CDC Bottle Assay. Based on these Assays, insecticide resistance in malaria vectors has been identified in at least 64 malaria-endemic countries worldwide, including 27 African countries, where it has been observed in major vector species of the $A n$. gambiae complex (An. arabiensis, An. gambiae s.s. and An. coluzzii) and An. funestus group [28-30]. The resistance has been mainly linked to the presence of either $k d r$ mutations (L1024F or L1014S alleles) or/and increased oxidase, esterase and glutathione S-transferase activities [31, 32]. It is noteworthy to mention that phenotypic bioassays are the definitive measure for resistance, though the detection of underlying mechanisms is also essential.

WHO tube assays performed during the current study revealed a remarkable reduction of mortality (59-76.5\%) and a moderate increase in knock-down times (less than 2.5 -fold) to DDT and pyrethroids in Pitoa. In other words, the significant decrease of mortality was not associated with a radical increase in knock-down times; this pattern of pyretroid resistance in An. gambiae s.l. from Pitoa was previously reported by Etang et al. [8]. Therefore, variations of knock-down times appear not to be a strong phenotypic indicator of DDT and pyrethroid resistance in Pitoa, when assessed by means of diagnostic concentrations. Such resistance pattern is likely specific to metabolic-based mechanisms of resistance in An. gambiae s.l. [8] rather than the $k d r L 1014$ mutations which usually result in both high increase of knock-down times and matching decrease of mortality rates [33]. Although the $k d r L 1014 F$ allele was recorded in the tested population of An. gambiae s.l. from Pitoa, the overall frequency was low (less than $5 \%$ ). This was the first report of the $k d r$ mutation in An. coluzzii from Pitoa, which is expected to contribute to resistance in addition to previously reported metabolic detoxification, suggesting multiple resistance profiles among the three sibling species. Chouaibou et al. [19] reported both $k d r L 1014 F$ and $L 1014 S$ alleles at very low frequencies $(1 / 45,1.1 \%)$ in An. gambiae s.s. specimens that survived insecticide exposure through WHO tube Assay with pyrethroids. From that time until 2011, no $k d r$ L1014 mutation has been reported in An. arabiensis from Pitoa.

The level of DDT and pyrethroid resistance recorded in the current study based on discriminative dosage and the overall $k d r L 1014 F$ frequency is classified as moderate, according to the stratification of Strode et al. (25-80\% mortality rate and $<25 \% k d r$ frequency) [34]. However, the dose response Assays with a range of deltamethrin concentrations revealed $\geq 500$ fold magnitude of resistance in the An. gambiae s.l. population from Pitoa, compared with the Kisumu susceptible An. gambiae s.s. strain. It has been demonstrated that "intensity Assays" using different multipliers of the discriminating dose that measure the strength of resistance, rather than Assays with discriminating doses, illustrates well the resistance selection and correlate better with control failure [35].

Considering the fact that pyrethroid insecticides have been commonly used in the Pitoa cotton areas, larvae of An. gambiae s.l. might be exposed to selection pressure in their breeding sites. Furthermore, several other factors might influence the intensity of selection and the development of resistance in the Pitoa, including the number of genes interacting to produce the resistant phenotype, the dominance relationship of the alleles as well as the size and proportion of the population affected by insecticide treatments as suggested by Chareonviriyaphap et al. [36]. Therefore, the observed variation in the frequencies of $k d r L 1014 F$ allele among adults of An. coluzzii, An. gambiae s.s., An. arabiensis might result from physiological or behavioural differences that cause An. gambiae s.s and An. coluzzii adults to be more extensively exposed to selective pressure from pyrethroids in LLINs and IRS, resulting in an increase of $k d r L 1014 F$ allelic frequency than $A n$. arabiensis. Indeed, An. arabiensis equally bites humans or animals depending on availability of the host [37]. In areas with universal coverage of LLINs or IRS, the high plasticity of biting habit of $A n$. arabiensis between human and alternative hosts might reduce the frequency of its contact with treated substrates and therefore exposure to insecticide selection pressure. Meanwhile, An. gambiae s.s. and An. coluzzii, which are more anthropophagic vectors [37], would mostly be exposed to contact with and selection by insecticide treated substrates when struggling to have human blood meals in the face of insecticide treatment.

In terms of operational implications of insecticide resistance, it is likely that $k d r$-based resistance exerts a differential impact on LLIN effectiveness compared to metabolic-based resistance as suggested by Strode et al. [34]. Metabolic-based resistance has been directly implicated in operational control failure of pyrethroids against An. funestus from South Africa [30]. In Pitoa, a set of constitutively over-expressed antioxidant genes and a single P450, CYP4G16, were associated with increased tolerance to deltamethrin in the An. arabiensis field population. These antioxidant genes include the superoxide dismutases SOD2 and SOD3, the glutathione S-transferase GSTS1 and the thioredoxin-dependent peroxidase TPX4 [32].

Indeed, metabolic-based resistance is likely the main DDT and pyrethroid resistance mechanisms in An. gambiae s.l. from Pitoa, since the $k d r$ L1014F allele, which is recessive, was found at low frequency and heterozygous 
state in the current study. $K d r$ is considered a relatively weak form of resistance compared to metabolic-based resistance, and it is usually only when $k d r$ occurs along with metabolic resistance that control fails [14, 30]. Nevertheless, the increase of $k d r$ frequency in addition to previously reported metabolic-based resistance and the remarkable resistance intensity recorded from the current study call attention to the risk of subsequent failure of conventional vector control interventions in Pitoa. Although new LifeNets were effective in killing this multiple resistant An. gambiae s.l. population, the subsequent decrease of bio-efficacy after nets were washed emphasizes the need for further investigations on the evolution of deltamethrin resistance in Pitoa during the coming years and the associated profile of LLINs bio-efficacy. Trials of new generations of LLINs which are combined with synergists (e.g. Piperonylbutoxide) to enhance their bio-efficacy against metabolic-based resistant mosquito populations should be encouraged in the Pitoa health district and other settings with a similar insecticide resistance pattern.

\section{Conclusions}

Data from the current study confirmed DDT and pyrethroid resistance in the An. gambiae s.l. population from Pitoa as previously revealed by the WHO standard susceptibility tests. Furthermore, the dose response test demonstrated a noteworthy magnitude of deltamethrin resistance in this vector population (i.e. $R R \geq 500$ fold increase), compared with the Kisumu susceptible reference strain. More interestingly, newly opened LifeNet LLINs were effective against this deltamethrin resistant mosquito population, although the residual bio-efficacy declined and fewer than $80 \%$ of mosquitoes were killed between 10 and 15 washes. The intensity of insecticide resistance and its impact on LLINs efficacy should therefore be regularly monitored in order to anticipate the replacement periods of LLINs or to choose appropriate LLINs in resistance areas.

\begin{abstract}
Abbreviations
DDT: dichloro-diphenyl-trichlorethane; DNA: deoxyribonucleic acid; HOLA: hot ligation oligonucleotide assay; $\mathrm{KD}_{60 \mathrm{~min}}$ : knock-down rate 60 minutes post exposure to insecticide treated net; $k d r$ : knock-down resistance; $\mathrm{KDT}_{50}$ : time of knock-down for $50 \%$ tested specimens; $\mathrm{KDT}_{50} \mathrm{R}$ : ratio times of knock-down for $50 \%$ between tested population and the Kisumu susceptible strain; KDT 95 : time of knock-down for $95 \%$ tested specimens; $L_{100}$ : lethal concentration for $100 \%$ specimens; $L C_{50}$ : lethal concentration for $50 \%$ specimens; LLINs: Long Lasting Insecticidal Nets; OCEAC: Organisation de Coordination pour la lutte contre les Endémies en Afrique Centrale;

PCR-RFLP: polymerase chain reaction-restricted fragment length polymorphism; PND: polypropylene net treated with deltamethrin; PRD: polyester net treated with deltamethrin; RR: ratio of mortality rates between tested population and the Kisumu susceptible strain; WHO: World Health Organization.
\end{abstract}

\section{Competing interests}

The authors declare that they have no competing interests.

\section{Authors' contributions}

$J \mathrm{E}, \mathrm{CP}$, and VC conceived and designed the study protocol. PM, AB and JE carried out field and laboratory assays. JE and PA analysed and interpreted data, and wrote the paper. MC and FC critically reviewed the manuscript. All the authors read and approved the final version of the manuscript.

\section{Acknowledgment}

The study was funded by Anopheles Biology and Control Network, with the support of Bayer Environmental Science. Authors are grateful to Mr. Feshu Nono Betrand for his assistance in statistical analysis.

\section{Author details}

'Institut de Recherche de Yaoundé (IRY), Organisation de Coordination pour la lutte contre les Endémies en Afrique Centrale (OCEAC), B.P. 288, Yaoundé, Cameroun. ${ }^{2}$ Faculty of Medicine and Pharmaceutical Sciences, University of Douala, P.O. Box 2701, Douala, Cameroon. ${ }^{3}$ Institut de Recherche pour le Développement (IRD) UMR224 MIVEGEC, 34394 Montpellier, France. ${ }^{4}$ Institut Pierre Richet (IPR), BP1500, Bouaké, Côte d'Ivoire. ${ }^{5}$ Ecole des Sciences de la Santé, Université Catholique d'Afrique Centrale, B.P. 1110, Yaoundé,

Cameroun. ${ }^{6}$ Centre de Recherche Entomologique de Cotonou (CREC), 01 B.P. 4414, Cotonou, Bénin. ${ }^{7}$ Institute of Tropical Medicine, Department Parasitology - Entomology, Nationalestraat 155, B-2000 Antwerpen, Belgium. ${ }^{8}$ University of Antwerp, Antwerp, Belgium. ${ }^{9}$ Department of Entomology, Faculty of Agriculture, Kasetsart University, Bangkok 10900, Thailand.

Received: 1 October 2015 Accepted: 2 March 2016

Published online: 08 March 2016

\section{References}

1. Mnzava AP, Knox TB, Temu EA, Trett A, Fornadel C, Hemingway J, et al. Implementation of the global plan for insecticide resistance management in malaria vectors: progress, challenges and the way forward. Mal J. 2015;14:173.

2. Glunt KD, Abílio AP, Bassat Q, Bulo H, Gilbert AE, Huijben S, Manaca MN, Macete E, Alonso P, Paaijmans KP. Long-lasting insecticidal nets no longer effectively kill the highly resistant Anopheles funestus of southern Mozambique. Malar J. 2015;14:298. doi:10.1186/s12936-015-0807-z.

3. IRmapper. www.irmapper.com. Accessed 2 Aug 2015.

4. Same-Ekobo A. Aspects épidémiologiques du paludisme au Cameroun. J Cam AcadSci. 2005;5(S1):3-16

5. MINSANTE. Plan stratégique National de lutte contre le paludisme au Cameroun 2011-2015. Cameroon: Ministry of Health; 2011.

6. Antonio-Nkondjo C, HinzoumbeKerah C, Simard F, Awono-Ambene P, Chouaibou M, Tchuinkam T, et al. Complexity of the malaria vectorial system in Cameroon: contribution of secondary vectors to malaria transmission. J Med Entomol. 2006;43(6):1215-21.

7. Etang J, Manga L, Chandre F, Guillet P, Fondjo E, Mimpfoundi R, et al. Insecticide susceptibility status of Anopheles gambiaes.I. (Diptera: Culicidae) in the Republic of Cameroon. J Med Entomol. 2003;40:491-7.

8. Etang J, Manga L, Toto JC, Guillet P, Fondjo E, Chandre F. Spectrum of metabolic-based resistance to DDT and pyrethroids in Anopheles gambiaes.l. populations from Cameroon. J VectorEcol. 2007:32:123-33.

9. Nwane P, Etang J, Chouaibou M, Toto JC, Kerah-Hinzoumbé C, Mimpfoundi $\mathrm{R}$, et al. Trends in DDT and pyrethroid resistance in Anopheles gambiaes.S. populations from urban and agro-industrial settings in southern Cameroon. BMC InfDis. 2009;9:163.

10. Nwane P, Etang J, Chouaibou M, Toto JC, Mimpfoundi R, Simard F. Kdr-based insecticide resistance in Anopheles gambiaes.s. populations in Cameroon: spread of the L1014F and L1014S mutations. BMC Research Notes. 2011;4:463.

11. Nwane P, Etang J, Chouaibou M, Toto JC, Koffi A, Mimpfoundi R, et al. Multiple insecticide resistance mechanisms in Anopheles gambiae s.l. populations from Cameroon, Central Africa. Parasites \& Vectors. 2013;6:41.

12. Kleinschmidt I, Mnzava AP, Kafy HT, Mbogo C, Bashir Al, Bigoga J, et al. Design of a study to determine the impact of insecticide resistance on malaria vector control: a multi-country investigation. Malar J. 2015;14:282.

13. Darriet F, N'guessan R, Koffi AA, Konan L, Doannio JMC, Chandre F, et al. Impact of the resistance to pyrethroids on the efficacy of impregnated bednets used as a means of prevention against malaria: results of the evaluation carried out with deltamethrin SC in experimental huts. Bull Soc Path Exoth. 2000;93(2):131-4. 
14. Henry MC, Assi SB, Rogier C, Dossou-Yovo J, Chandre F, Guillet P, et al. Protective efficacy of lambda-cyhalothrin treated nets in Anopheles gambiae pyrethroid resistance areas of Cote d'Ivoire. Am J Trop Med Hyg. 2005:73(5):859-64.

15. Damien GB, Djènontin $A$, Rogier $C$, Corbel V, Bangana SB, Chandre $F$, et al. Malaria infection and disease in an area with pyrethroid-resistant vectors in southern Benin. Malar J. 2010;9(1):380

16. N'Guessan R, Corbel V, Akogbeto M, Rowland M. Reduced efficacy of insecticide-treated nets and indoor residual spraying for malaria control in pyrethroid resistance area, Benin. Emerg Infect Dis. 2007;13:199-206.

17. Asidi A, N'Guessan R, Akogbeto M, Curtis C, Rowland M. Loss of household protection from use of insecticide-treated nets against pyrethroid-resistant mosquitoes, Benin. Emerg Infect Dis. 2012;18:1101-6.

18. Etang J, Chouaibou M, Toto JC, Faye O, Manga L, Samè-Ekobo A, Awono-Ambene $\mathrm{P}$, Simard F. A preliminary test of the protective efficacy of Permethrin-treated bed nets inan area of Anopheles gambiaemetabolic resistance to pyrethroids in NorthCameroon. Trans R Soc Trop Med Hyg. 2007;101:881-4.

19. Chouaïbou M, Etang J, Brévault T, Nwane P, Kérah HC, Mimpfoundi R, Simard F. The dynamics of insecticide resistance in the malaria vector Anopheles gambiaes.l. from an area of extensive cotton cultivation in Northern Cameroon. Trop Med Int Health. 2008;13(4):476-86.

20. Gillies MT, De Meillon B. The Anophelinae of Africa south of the Sahara. Johannesburg: Publications of the South African Institute for Medical Research; 1968. p. no. 54.

21. Gillies MT, Coetzee M. A supplement to the Anophelinae of Africa south of the Sahara. Johannesburg: Publications of the South African Institute for Medical Research; 1968. p. no. 55.

22. WHO. Test procedures for insecticide resistance monitoring in malaria vector mosquitoes. World Health Organization; 2013.

23. WHO. Guidelines for laboratory and field testing of long-lasting insecticidal nets. Geneva: World Health Organization; 2013. WHO/HTM/NTD/WHOPES/20131.

24. Collins FH, Mendez MA, Razmussen MO, Mehaffey PC, Besansky NJ, Finnerty VA. Ribosomal RNA gene probe differentiates member species of Anopheles gambiae complex. Am J Trop Med Hyg. 1987;37:37-41.

25. Fanello C, Santolamazza F, DdellaTorré A. Simultaneous identification of species and molecular forms of the Anopheles gambiae complex by PCR-RFLP. Med Vet Entomol. 2002;16:461-4.

26. Lynd A, Ranson H, McCall PJ, Randle NP, Black IV WC, Walker ED, et al. A simplified high-throughput method for pyrethroid knock-down resistance (kdr) detection in Anopheles gambiae. Malaria J. 2005;4:16.

27. Finney DJ. Probit analysis. 3rd ed. Cambridge: Cambridge University Press. University of Oxford, Oxford, England; 1971. p. no. 333.

28. Corbel V, N'Guessan R. Distribution, mechanisms, impact and management of insecticide resistance in malaria vectors: a pragmatic review. In: Manguin S, editor. Anopheles mosquitoes-New insights into malaria vectors. 2013. p. 579-633. doi:10.5772/56117. In Tech.

29. Ranson H, N'Guessan R, Lines J, Moiroux N, Nkuni Z, et al. Pyrethroid resistance in African anopheline mosquitoes: what are the implications for malaria control? Trends Parasitol. 2011;27:91-8

30. Hargreaves K, Koekemoer LL, Brooke BD, Hunt RH, Mthembu J, Coetzee M. Anopheles funestus resistant to pyrethroid insecticides in South Africa. Med Vet Entomol. 2000;14:181-9.

31. Santolamazza F, Calzetta M, Etang J, Barrese E, Dia I, Caccone A, et al. Distribution of knockdown resistance mutations in Anopheles gambiae (Diptera:Culicidae) molecular forms in West and Central-West Africa. Mal J. 2008;7:74.

32. Müller $\mathrm{P}$, Chouaibou M, Pignatelli $\mathrm{P}$, Etang J, Walker ED, Donnelly MJ, et al. Pyrethroid tolerance is associated with elevated expressionof antioxidants and agricultural practice in Anophelesarabiensissampled from an area of cotton fields in NorthernCameroon. Mol Ecol. 2008. doi:10.1111/j.1365-294x.200703617.x.

33. Chandre F, Frederic D, Sylvie M, Cecile B, Carnevale P, Guillet P. Pyrethroid cross resistance spectrum among populations of Anopheles gambiaes.s. from Côte d'Ivoire. J Am Cont Ass Inc. 1999;15(1):53-9.

34. Strode C, Donegan S, Garner P, Enayati AA, Hemingway J. The impact of pyrethroid resistance on the efficacy of insecticide-treated Bed nets against African anopheline mosquitoes: systematic review and meta-analysis. PLoS Med. 2014;11:3-e1001619. doi:10.1371/journal.pmed.1001619.

35. Bagi J, Grisales N, Corkill R, Morgan JC, N'Falé S, Brogdon W, Ranson H. When a discriminating dose assay is not enough: measuring the intensity of insecticide resistance in malaria vectors. Malar J. 2015:14:210. doi:10.1186/s12936-015-0721-4
36. Chareonviriyaphap $T$, Rongnoparut $P$, Juntarumporn $P$. Selection for pyrethroid resistance in a colony of Anopheles minimus species A, malaria vector in Thailand. J Vector Ecol. 2002;27:222-9.

37. Busula AO, Takken W, Loy DE, Hahn BH, Mukabana WR, Verhulst NO. Mosquito host preferences affect their response to synthetic and natura odour blends. Malaria J. 2015;14:133. doi:10.1186/s12936-015-0635-1.

\section{Submit your next manuscript to BioMed Central and we will help you at every step:}

- We accept pre-submission inquiries

- Our selector tool helps you to find the most relevant journal

- We provide round the clock customer support

- Convenient online submission

- Thorough peer review

- Inclusion in PubMed and all major indexing services

- Maximum visibility for your research

Submit your manuscript at www.biomedcentral.com/submit
) Biomed Central 